\section{CORONARY FLOW RESERVE AFTER ISCHEMIA AND REPERFUSION OF THE ISOLATED HEART}

\section{Divergent results with crystalloid versus blood perfusion}

\footnotetext{
From the Divisions of Cardiac and Thoracic Surgery $^{a}$ and Hypertension Research, ${ }^{b}$ Henry Ford Hospital, Detroit, Mich.

Presented in part at the Forty-ninth Annual Sessions of the Forum on Fundamental Surgical Problems, 1993 Clinical Congress, San Francisco, Calif., October 10-15.

Received for publication March 10, 1994.

Accepted for publication July 22, 1994.

Address for reprints: Norman A. Silverman MD, Division Head, Cardiac and Thoracic Surgery, Henry Ford Hospital, 2799 W. Grand Blvd., Detroit, MI 48202.

Copyright $(1995$ by Mosby-Year Book, Inc.

$0022-5223 / 95 \$ 3.00+0 \quad \mathbf{1 2 / 1 / 5 9 3 6 7}$
}

Mechanical function and coronary hemodynamics were assessed in 73 isolated rabbit hearts randomly subjected to $0,10,20,30$, or 45 minutes of $37^{\circ} \mathrm{C}$ global ischemia and 45 minutes of reperfusion in either a modified Krebs buffer or homologous blood-perfused Langendorff mode ( $n=7$ to 9 hearts per group). Isovolumic developed pressure, resting coronary flow, and response to endotheliumdependent (bradykinin) and -independent (nitroglycerin) agonists were quantitated at defined preload and heart rate. Perfusate did not influence systolic performance, which was impaired after 30 minutes of ischemia and fell to $64 \%$ to $72 \%$ of preischemic values after 45 minutes of ischemia $(p<0.05)$. However, basal coronary flow was at least sixfold greater in crystalloid-perfused hearts. Moreover, coronary hyperemia $(p<0.05)$ persisted for Krebs-perfused hearts subjected to all but the longest ischemic interval. After equilibration, all postischemic blood-perfused hearts had basal flow unchanged from before ischemia. Bradykinin and nitroglycerin induced similar increases in coronary flow for each group before and after each ischemia interval. However, the magnitude of this increase was greater in bloodperfused hearts $(p<0.01)$ and was not attenuated by the ischemic times encompassed in this protocol. In contrast, endothelium-dependent and -independent coronary flow reserve was abolished after 20 minutes of ischemia or longer in Krebs-perfused hearts. These data suggest that the unphysiologic resting flow patterns of crystalloid-perfused isolated hearts obfuscate interpretation of the interaction between coronary flow reserve and ischemic injury. (J THORAC CARDIOVASC SURG 1995;109:466-72)

Quanmei Deng, MD, ${ }^{\mathrm{a}}$ Alfonso G. Scicli, PhD, ${ }^{\mathrm{b}}$ Carol Lawton, BS, ${ }^{\mathrm{a}}$ and Norman A. Silverman, MD, ${ }^{\text {a }}$ Detroit, Mich.
$T^{n}$ he isolated, perfused, small animal heart has been a standard experimental model for quantitating the consequences of ischemia and reperfusion. Although the focus of previous investigations has historically been on myocyte viability as assessed by postischemic ventricular performance and cardiac metabolism, the coronary arterial smooth muscle and the endothelium with its paracrine function are now recognized as separate and important considerations in formulating strategies to prevent ischemia-reperfusion injury. ${ }^{1-2}$ Crystalloid-perfused heart preparations have the advantages of lower cost, precision of formulation, simplicity, and avoidance of thrombus formation. However, clinical relevance will always be questionable because the roles of erythrocytes, platelets, and leukocytes in oxidative stress and reperfusion injury are excluded. Also, the lack of plasma proteins results in osmotic and oncotic inadequacies and the baseline high oxygen tension and coronary flow rate are unphysiologic. ${ }^{3}$

The present study was designed to quantitate and compare perturbations in basal coronary flow and coronary hemodynamics stimulated by endothelium-dependent and endothelium-independent agonists after progressively severe, reversible ischemic insults in crystalloid- and blood-perfused isolated hearts. These observations suggest that the unphysiologic resting flow patterns attendant to asanguineous perfusates obfuscate interpretation of coronary flow reserve after ischemic injury.

\section{Materials and methods}

Langendorff perfusion. The details of the blood-perfused ex vivo preparation have been previously described. ${ }^{4}$ All animals received humane care in compliance with the "Principles of Laboratory Animal Care" formulated by the National Society for Medical Research and the "Guide for the Care and Use of Laboratory Animals" prepared by the National Academy of Sciences and published by the National Institutes of Health (NIH Publication No. 80-23, revised 1978). Adult New Zealand White rabbits $(2.5$ to $3.8 \mathrm{~kg})$ were anesthetized, heparinized, a tracheostomy performed, and the lungs ventilated with room air. The abdomen of the blood-donor rabbit was opened and the abdominal aorta was cannulated with an 18-gauge ball-end needle for blood collection. The thorax 
Table I. Systolic performance during the experimental protocol

\begin{tabular}{|c|c|c|c|c|c|}
\hline & \multirow[b]{2}{*}{ Before ischemia } & \multicolumn{4}{|c|}{ After ischemia } \\
\hline & & $10 \mathrm{~min}$ & $20 \mathrm{~min}$ & $30 \mathrm{~min}$ & $45 \mathrm{~min}$ \\
\hline \multicolumn{6}{|c|}{$\mathrm{DP}(\mathrm{mm} \mathrm{Hg})$} \\
\hline Krebs & $110 \pm 6$ & $117 \pm 3$ & $107 \pm 10$ & $79 \pm 6^{*}$ & $70 \pm 8^{*}$ \\
\hline Blood & $97 \pm 4$ & $119 \pm 11$ & $84 \pm 11$ & $72 \pm 8^{*}$ & $70 \pm 9^{*}$ \\
\hline \multicolumn{6}{|c|}{$+\mathrm{dp} / \mathrm{dt}(\mathrm{mm} \mathrm{Hg} \mathrm{sec}-1)$} \\
\hline Krebs & $1556 \pm 133$ & $1662 \pm 77$ & $1443 \pm 236$ & $1228 \pm 150$ & $977 \pm 180^{*}$ \\
\hline Blood & $1461 \pm 99$ & $1401 \pm 109$ & $1302 \pm 299$ & $1262 \pm 155$ & $1052 \pm 286^{*}$ \\
\hline
\end{tabular}

Values are mean \pm standard error of the mean; $n=7$ to 9 hearts per group; $D P$, Developed pressure.

${ }^{*} p<0.05$ versus before ischemia.

and abdomen of the heart-donor rabbit were opened. Both the abdominal aorta and ascending aorta were cannulated in situ and retrograde perfusion was initiated before the heart was harvested as blood was withdrawn and added to the venous reservoir. The pulmonary artery was cannulated for collection and measurement of coronary effluent. The heart was then harvested and mounted on the siliconized glass perfusion column. Normothermic retrograde perfusion was initiated at $50 \mathrm{~mm} \mathrm{Hg}$ for 5 minutes and then increased to $80 \mathrm{~mm} \mathrm{Hg}$ by varying the speed of the roller pump. The blood perfusate was oxygenated by bubbling the reservoir beaker with a mixture of $95 \%$ oxygen and $5 \%$ carbon dioxide to maintain oxygen tension between 120 and 200 torr and carbon dioxide tension between 35 and 45 torr. The $\mathrm{pH}$ was corrected to 7.35 to 7.45 with sodium bicarbonate. Crystalloid-perfused hearts were similarly cannulated and perfused in situ before being mounted to minimize ischemia. Hearts did not stop beating in the interval between harvesting and mounting. The modified Krebs buffer solution was bubbled with a $95 \%$ oxygen and $5 \%$ carbon dioxide mixture. The blood perfusion was a recirculating system whereas the Krebs perfusate was not recirculated.

All hearts were immersed in a water-jacketed organ chamber to maintain a temperature of $37^{\circ} \mathrm{C}$. During stabilization after retrograde perfusion was established, the venae cavae were ligated, the sinus node crushed, the lungs removed, and all hearts were instrumented in the following manner. The left atrium was opened and a size 10 intraventricular latex balloon (Hugo Sachs Elektronik, March-Hugstetten, Germany) secured below the mitral anulus. A $1 \mathrm{~mm}$ diameter plastic catheter effected left ventricular venting. A saline filled Tygon microbore tube (Norton Performance Plastics, Wayne, N.J.) connected the balloon to a P23XL transducer (Viggo-Spectramed, Inc., Sacramento, Calif.) for measurement of left ventricular pressure, developed pressure, and the rate of positive and negative pressure development $( \pm \mathrm{dp} / \mathrm{dt})$. Pressure tracings were recorded on an eight-channel recorder (RS 3800, Gould, Inc., Cleveland, Ohio). Ventricular electrodes were attached and the hearts paced at 180 beats/ min. For the purpose of drug testing, the bolus infusion was administered through a sidearm line to the main perfusion line.

Experimental protocol. Hearts were perfused with either oxygenated Krebs buffer or homologous blood and randomly subjected to $10,20,30$, or 45 minutes of $37^{\circ} \mathrm{C}$ global ischemia effected by stopping the perfusion pump. Control hearts were studied without antecedent ischemia. After a 45-minute equilibration time with retrograde perfusion, baseline isovolumic systolic function and coronary flow were quantitated at the fixed paced rate and an end-diastolic pressure of $10 \mathrm{~mm} \mathrm{Hg}$. Endothelium-dependent coronary flow reserve was quantitated after bolus infusion of $1 \mathrm{ml} 10^{-6}$ molar bradykinin. A 12-minute washout period allowed resting coronary flow to return to baseline before endothelium-independent coronary flow reserve was quantitated by bolus infusion of $500 \mu \mathrm{g}$ nitroglycerin. Hearts were then removed from the perfusion column, excess fluid gently removed, and the hearts were placed in a drying oven for 48 hours. At that time, hearts were removed and weighed. Data were obtained from seven to nine hearts for each perfusate as controls and for each ischemic interval.

Systolic function. The intraventricular balloon was filled with a volume of saline to produce an end-diastolic pressure of $10 \mathrm{~mm} \mathrm{Hg}$. Isovolumic developed pressure at this preload (millimeters of mercury) was calculated as the difference between peak left ventricular systolic pressure and this diastolic pressure.

Coronary flow. Three-minute timed collection of coronary venous effluent was made at each sampling interval. Results were expressed as milliliters per minute per gram dry weight of the heart.

Statistical methods. Data are presented as mean \pm standard error of the mean. Paired observations were compared by Student's $t$ test. Repeated observations were compared by analysis of variance with Tukey's method used to adjust for multiple tests. Differences were considered significant if $p$ was $\leq 0.05$.

\section{Results}

Systolic function (Table I). Krebs-perfused hearts had no decrement in developed pressure after the 10 - and 20 -minute ischemic intervals. However, the $72 \%$ and $64 \%$ returns of preischemic developed pressure after 30 and 45 minutes of ischemia were depressed ( $p<0.05$ for each) compared with control values. In blood-perfused hearts, there was an incremental decrease in developed pressure after 20 minutes and longer ischemic inter- 


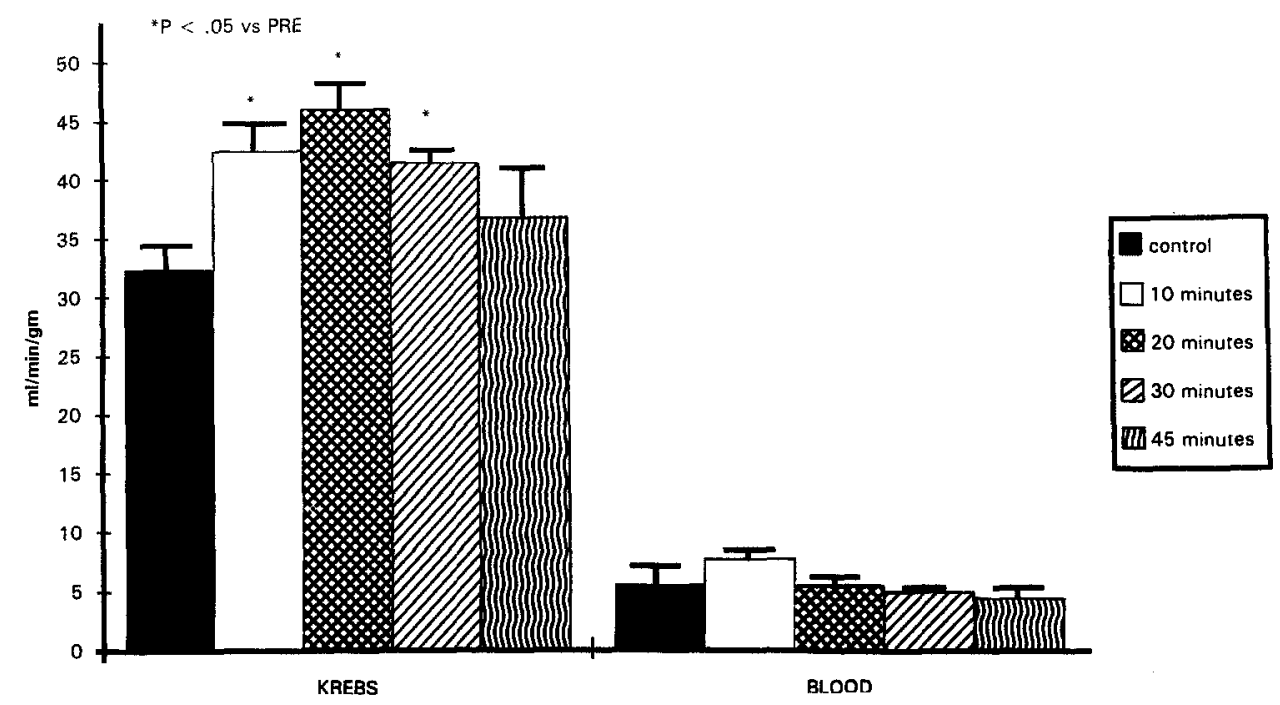

Fig. 1. Comparison of unstimulated coronary flow in the experimental groups. With Krebs perfusate, $28 \%$ to $42 \%$ increases were noted following after 10 to 30 minutes of ischemia, whereas with blood perfusate no significant hyperemia was noted 45 minutes after the progressive ischemic intervals.

Table II. Coronary flow patterns during the expermental protocol

\begin{tabular}{|c|c|c|c|c|c|}
\hline & \multirow[b]{2}{*}{ Before ischemia } & \multicolumn{4}{|c|}{ After ischemia } \\
\hline & & $10 \mathrm{~min}$ & $20 \mathrm{~min}$ & $30 \mathrm{~min}$ & $45 \mathrm{~min}$ \\
\hline \multicolumn{6}{|c|}{$\begin{array}{l}\text { Basal CF (ml/min per gram } \\
\text { dry weight) }\end{array}$} \\
\hline Krebs & $32.4 \pm 2.1$ & $42.5 \pm 2.3^{*}$ & $46.0 \pm 2.1^{*}$ & $41.4 \pm 1.0^{*}$ & $36.8 \pm 4.2$ \\
\hline Blood & $5.5 \pm 1.6$ & $7.7 \pm 0.8$ & $5.4 \pm 0.7$ & $4.9 \pm 0.2$ & $4.4 \pm 0.9$ \\
\hline \multicolumn{6}{|c|}{$\begin{array}{l}\text { Increase in } \mathrm{CF}(\mathrm{ml} / \mathrm{min} \text { per } \\
\text { gram dry weight) }\end{array}$} \\
\hline Krebs-BK & $7.5 \pm 1.5$ & $5.8 \pm 1.6$ & $3.1 \pm 1.9^{*}$ & $0.2 \pm 0.2^{*}$ & $1.9 \pm 0.6^{*}$ \\
\hline Blood-BK & $4.4 \pm 1.4$ & $7.4 \pm 0.99$ & $6.6 \pm 1.3$ & $5.9 \pm 1.4$ & $6.0 \pm 1.2$ \\
\hline Krebs-NTG & $6.5 \pm 1.3$ & $3.8 \pm 2.2$ & $1.6 \pm 1.1$ & $0.3 \pm 0.5^{*}$ & $2.6 \pm 0.6^{*}$ \\
\hline Blood-NTG & $4.2 \pm 0.8$ & $4.5 \pm 1.1$ & $3.4 \pm 0.8$ & $3.6 \pm 0.5$ & $3.8 \pm 1.1$ \\
\hline
\end{tabular}

Values are mean \pm standard error of the mean; $n=7$ to 9 hearts per group; $C F$, coronary flow; $B K$, bradykinin 1 nmol; $N T G, 500 \mu \mathrm{g}$ nitroglycerin. ${ }^{*} p<0.05$ versus before ischemia.

vals that reached significance after 30 and $45 \mathrm{~min}$ utes of ischemia with reperfusion $(74 \%$ and $72 \%$, respectively, $p<0.05$ versus control for each). A similar pattern of maximum $+\mathrm{dp} / \mathrm{dt}$ was noted, declining to $63 \%$ and $72 \%$ of control in Krebs- and blood-perfused hearts, respectively, after 45 minutes of ischemia ( $p<0.05$, each group). No difference in the two parameters of systolic function was noted in comparing Krebs- and blood-perfused hearts before and after each ischemic interval. Because heart rate was fixed by pacing, the mechanical determinants of coronary flow were comparable in the two groups throughout the experimental protocol.

Basal coronary flow (Table II, Fig. 1). In Krebsperfused hearts, control unstimulated coronary flow ranged between 21.3 and $48.2 \mathrm{ml} / \mathrm{min}$ per gram dry weight whereas blood-perfused hearts had coronary flow between 3.6 and $8.2 \mathrm{ml} / \mathrm{min}$ per gram dry weight. After ischemia and equilibration, coronary hyperemia persisted for Krebs-perfused hearts subjected to all but the longest ischemic interval $(p<$ 0.05 versus control for 10,20 , and 30 minutes of ischemia, respectively). When measured after the 45-minute equilibration period, unstimulated coronary flow after all ischemic intervals in bloodperfused hearts was unchanged from preischemic flow. A comparison of the groups at each study interval showed that coronary flow in Krebs-perfused hearts was 5.5- to 8.5-fold greater than in blood-perfused hearts ( $p<0.001$, all intervals). 


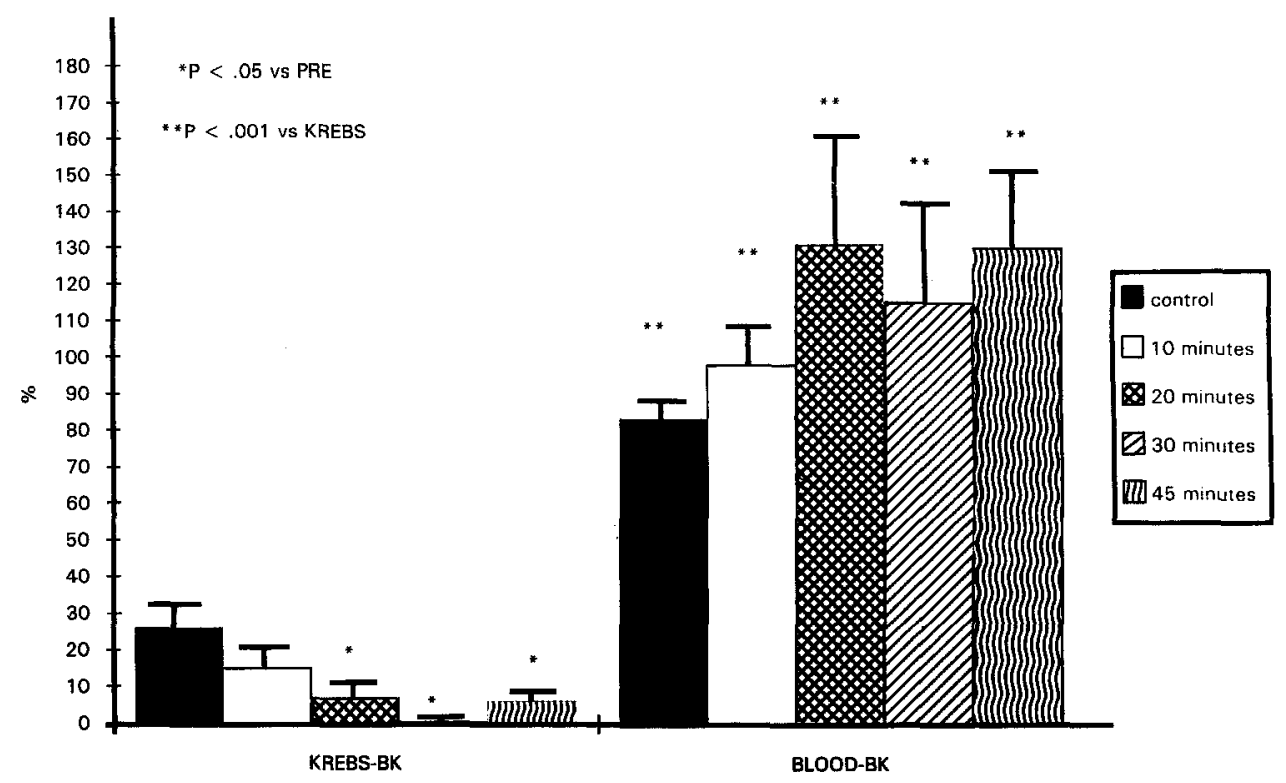

Fig. 2. Comparison of endothelium-dependent coronary flow reserve in the experimental groups quantitated by percentage increase over basal flow in response to bradykinin $(B K, 1 \mathrm{nmol})$.

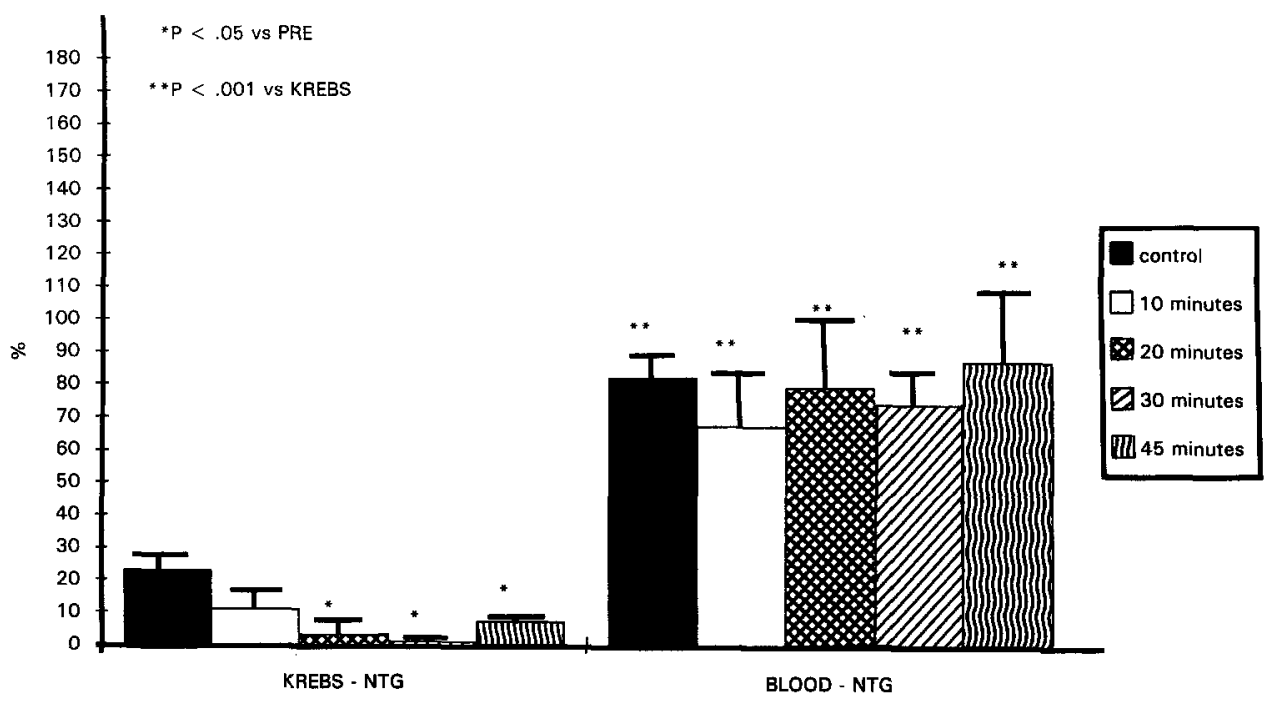

Fig. 3. Comparison of endothelium-independent coronary flow reserve in the experimental groups quantitated by percentage increase over basal flow in response to nitroglycerin $(N T G, 500 \mu \mathrm{g})$.

Coronary flow reserve (Table II, Figs. 2 to 4 ). Before ischemia, bradykinin and nitroglycerin increased coronary flow by $7.5 \pm 1.5$ and $6.5 \pm 1.3$ $\mathrm{ml} / \mathrm{min}$ per gram dry weight in Krebs-perfused hearts and $4.4 \pm 1.4$ and $4.2 \pm 0.8 \mathrm{ml} / \mathrm{min}$ per gram dry weight in blood-perfused hearts. The percentage increase over unstimulated coronary flow was almost identical for each agonist within each group, but the magnitude of this increase was almost fourfold greater with blood perfusion $(p<0.001)$. In
Krebs-perfused hearts, the responses to bradykinin and nitroglycerin were comparable after each of the ischemic intervals. The percentage increase over unstimulated flow was less than 10 minutes of ischemia, and no significant augmentation in coronary flow was inducible after the longer ischemic intervals. In contrast, the $98 \%$ to $130 \%$ and $67 \%$ to $87 \%$ increases in coronary flow stimulated by bradykinin and nitroglycerin, respectively, after graded ischemic insults in blood-perfused hearts did not 


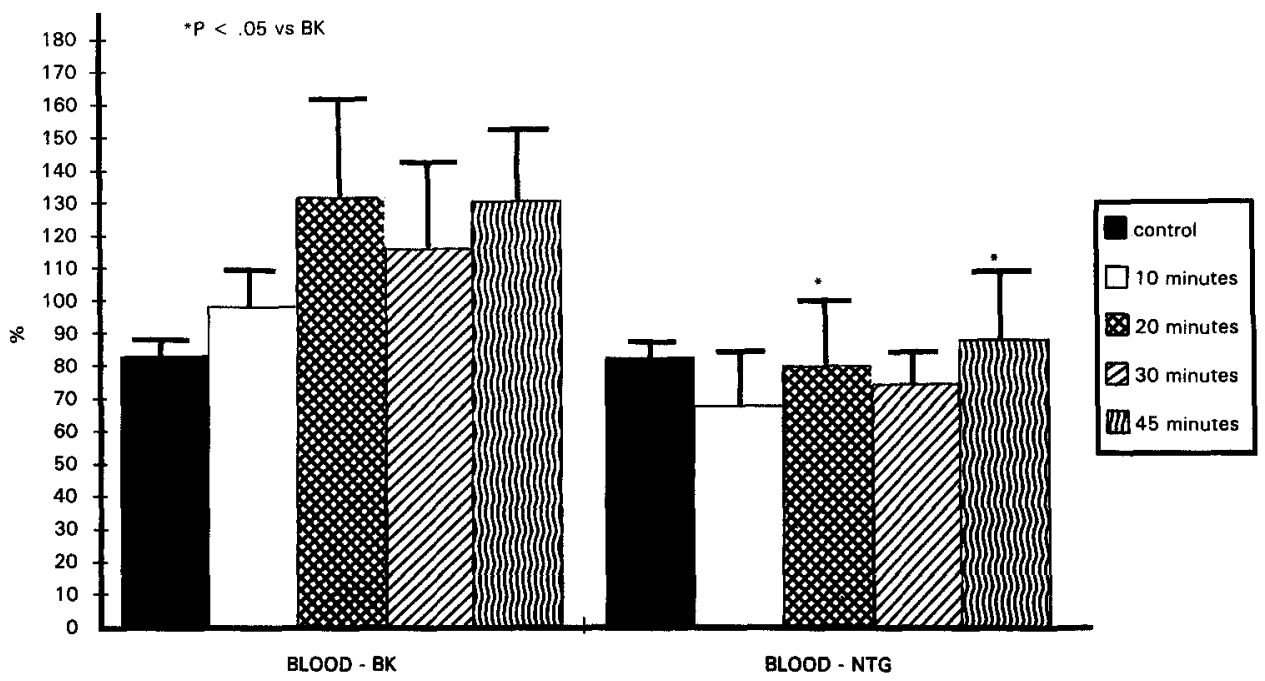

Fig. 4. Comparison of endothelium-dependent versus endothelium-independent coronary flow reserve in blood-perfused hearts quantitated by percentage increase over basal flow in response to bradykinin ( $B K$, $1 \mathrm{nmol})$ and nitroglycerin $(N T G, 500 \mu \mathrm{g})$.

differ from preischemic coronary flow reserve. The response to a given agonist was unaffected by ischemic time; however, for ischemic durations of 20 minutes or longer, the magnitude of the response to bradykinin was greater than that to nitroglycerin $(p<0.05)$.

Myocardial water content. For Krebs-perfused hearts, control myocardial water content was $85.9 \% \pm 1.3 \%$. After the various ischemic intervals, heart water content ranged from $85.4 \%$ to $86.5 \%$ and did not differ from control or in relation to ischemic interval. Blood-perfused hearts had $80.4 \%$ $\pm 0.9 \%$ water content before ischemia, and after the protocol intervals water content ranged from $78.1 \%$ to $81.3 \%$, again not different from control or in relation to ischemia. However, the Krebs-perfused hearts had significantly more water content than blood-perfused hearts at each study interval $(p<$ 0.01 , each interval).

\section{Discussion}

Conceptually, we have performed an experiment in an isolated small animal heart preparation to quantitate derangements in resting coronary flow and stimulated endothelium-dependent and endothelium-independent coronary flow reserve to incrementally more severe ischemic insults. We compared the relative susceptibility to unprotected, global normothermic ischemia of these coronary flow patterns with isovolumic systolic performance. Given an identical experimental protocol, implica- tions from the data generated depend on whether the heart was perfused with homologous blood or an asanguineous solution. In a blood-perfused heart, systolic performance was compromised, but the response to bradykinin and nitroglycerin was unaffected even after the longest ischemic interval, suggesting resistance of the endothelium and coronary arterial smooth muscle to ischemia-reperfusion injury. In contrast, in Krebs-perfused hearts, the response to these agonists was blunted before a decrement in systolic function was noted. Does this imply that the coronary vasculature including the endothelium is more susceptible than the myocyte to deprivation of flow? Alternatively, does the high shear stress of the unphysiologic resting coronary flow in the crystalloid-perfused heart result in almost maximal relaxation of arterial smooth muscle mediated by endothelium-derived relaxing factor (EDRF), thus precluding an incremental effect when an exogenous agonist is infused?

When conflicting interpretations exist, confirmation of alternative explanations is sought in the literature. Unfortunately, the various methods for quantitating coronary artery endothelial cell and smooth muscle dysfunction after ischemia may further obfuscate rather than clarify the issue. ${ }^{5-8}$ For instance, "endothelial function" can be tested in vivo by infusion of agonists to stimulate endothelial muscarinic, serotonergic, purinergic, thrombin, or vasopressinergic receptor-mediated EDRF release. EDRF causes relaxation of the underlying smooth muscle by a mechanism 
that involves cyclic guanosine monophosphate-dependent protein phosphorylation. What is usually measured is a change in coronary flow or resistance resulting from this vasodilation. Differences in dosage, agonist, and species make strict comparison difficult. Alternatively, ex vivo methods use harvested rings of epicardial vessels. Comparative arterial vascular responsiveness, that is, force of contraction, is quantitated by adding agonists to the bath with the endothelium intact or denuded and/or in the resting state or preconstricted. The lack of "gold standards" applicable to all laboratories is evident. Moreover, these ex vivo tests of vascular responsiveness are performed in epicardial conductance vessels, whereas the effects of an in vivo infusion of vasoactive agent are more likely at the coronary arteriolar level.

Several studies, mostly involving isolated canine epicardial coronary arterial rings, suggest that brief ischemia results in vascular endothelial dysfunction. Gross and colleagues ${ }^{9}$ showed attenuated endothelium-dependent responses after repetitive 5-minute occlusions but unaffected response to endotheliumindependent agonists. After 15 minutes of ischemia, Headrick, Angello, and Berne ${ }^{10}$ showed injury to the large coronary conduit vessels and Dauber and coworkers $^{11}$ also showed concomitant microvascular damage. The time course of recovery of this "stunned" endothelial function has been assessed by Kim and colleagues. ${ }^{12}$ After 15 minutes of ischemia, the impaired vasodilatory response to acetylcholine required 90 minutes to return to baseline. The response to nitroprusside was unaffected by their protocol. In vitro endothelium-dependent vasodilatory responses were blunted after 10 minutes of ischemia, abolished after 20 minutes, and required 120 minutes of reperfusion to recover. In contrast, the in vivo study of McFalls and associates ${ }^{13}$ found no diminution in endothelium-dependent coronary flow reserve of swine after 10 minutes of ischemia.

More easily comparable to the present study is the work of Hashimoto and coworkers. ${ }^{14}$ In crystalloidperfused isolated rabbit hearts subjected to $30 \mathrm{~min}-$ utes of ischemia and 45 minutes of reperfusion, serotonin increased coronary flow $30.5 \%$ before ischemia and only $13.4 \%$ after ischemia, whereas the smooth muscle response to adenosine was unaffected. Although postischemic systolic dysfunction was comparable to our 30-minute ischemic groups, the difference in endothelium-dependent and -independent flow reserve could be attributable to different agonists and lower basal flow rates. Similarly, Tsao and Lefer ${ }^{15}$ subjected isolated, crystalloid- perfused rat hearts to 30 minutes of global ischemia and found impaired relaxation to an alternative endothelium-dependent vasodilator, acetylcholine, after 20 minutes of reperfusion. No change in responsiveness to nitroglycerin was noted. However, unlike the present study, flow patterns to these agonists were quantitated after preconstriction of the coronary vasculature by U4619.

A detailed review of endothelial and arterial smooth muscle function after 1 hour of ischemia or more is not applicable to the presented data. However, conflicting results are reported that parallel the information available for "reversible ischemic" insults, that is, divergence of endothelium-dependent and -independent responses and actual existence of an injury. Representative of these controversies are the observations by Pearson, Schaff, and Vanhoutte, ${ }^{16,17}$ showing persistence of altered serotonergic and purinergic receptor activity 1 month after a 1-hour ischemic interval. Dignan and associates $^{18}$ found no vascular injury after 2 hours of ischemia, and Viehman and coworkers ${ }^{19}$ had to extend ischemia to 3 hours to demonstrate impaired vasoreactivity.

This study has several limitations. Admittedly, this is a descriptive and not a mechanistic protocol and therefore the questions posed in the first paragraph of the discussion are not answered. The relative contributions of altered viscosity, oxygen tension, formed blood cell elements, and wall shear stress were not analyzed. The crystalloid-perfused studies were purposely performed in a "standard" manner to compare with a more physiologic blood perfusate, and no attempt was made to control for the aforementioned variables. Second, the concentrations of agonist actually presented to the endothelial receptor may have been lower in the crystalloid-perfused hearts because of the increased resting coronary flow. However, preliminary doseresponse curves (data not shown) in both crystalloid and blood perfusates showed maximal response to the concentration of agonist infused by bolus injection. Therefore, we support the quantitative validity of these observations and propose that despite the simplicity of a crystalloid-perfused heart model, it is inappropriate for assessment of the interaction between coronary flow reserve and ischemic injury.

\section{REFERENCES}

1. $\mathrm{Ku} \mathrm{DD}$. Coronary vascular reactivity after acute myocardial ischemia. Science 1982;218:576-8.

2. VanBenthuysen KM, McMurtry IF, Horwitz LD. 
Reperfusion after acute coronary occlusion in dogs impairs endothelium-dependent relaxation to acetylcholine and augments contractile reactivity in vitro. $\mathbf{J}$ Clin Invest 1987;79:265-74.

3. Qui Y, Hearse DJ. Comparison of ischemic vulnerability and responsiveness to cardioplegic protection in crystalloid-perfused versus blood-perfused hearts. J Thorac Cardiovasc Surg 1992;103;960-8.

4. Deng Q, Scicli AG, Lawton C, et al. A simplified blood-perfused isolated heart preparation. J THORAC CARDIOVASC SuRg [In press].

5. Furchgott RF. Role of endothelium in responses of vascular smooth muscle. Circ Res 1983;53:557-73.

6. Vanhoutte PM, Shimokawa H. Endothelium-derived relaxing factor and coronary spasm. Circulation 1989; $80: 1-9$.

7. Vanhoutte PM. Other endothelium-derived vascular factors. Circulation 1993;87(Suppl):V9-17.

8. Meredith IT, Yeung AC, Weidinger FF, et al. Role of impaired endothelium-dependent vasodilation in ischemic manifestations of coronary artery disease. Circulation 1993;87(Suppl):V56-66.

9. Gross GJ, O'Rourke ST, Pelc LR, et al. Myocardial and endothelial dysfunction after multiple brief coronary occlusions: role of oxygen radicals. Am J Phsyiol 1992;263:1703-9.

10. Headrick JP, Angello DA, Berne RM. Effects of brief coronary occlusion and reperfusion on coronary artery reactivity. Circulation 1990;82:2163-9.

11. Dauber IM, VanBenthuysen KM, McMurtry IF, et al. Functional coronary microvascular injury evident as increased permeability due to brief ischemia and reperfusion. Circ Res 1990;66:986-98.
12. Kim YD, Foinsgaard JS, Heim KF, et al. Brief ischemia-reperfusion induces stunning of endothelium in canine coronary artery. Circulation 1992;85: 1473-82.

13. McFalls EO, Duncker DJ, Krams R, et al. Endothelium dependent vasodilation following brief ischemia and reperfusion in anesthetized swine. Cardiovasc Res 1991;25:659-65.

14. Hashimoto K, Pearson P, Schaff HV, et al. Endothelial cell dysfunction after ischemic arrest and reperfusion: a possible mechanism of myocardial injury during reflow. J THORAC CARDIOVASC SURG 1991;102:68894.

15. Tsao PS, Lefer AM. Time course and mechanism of endothelial dysfunction in isolated ischemic and hypoxic perfused rat hearts. Am J Physiol 1990;28: $1660-6$.

16. Pearson PJ, Schaff HV, Vanhoutte PM. Acute impairment of endothelium-dependent relaxations to aggregating platelets after reperfusion injury in canine coronary arteries. Circ Res 1990;67:385-93.

17. Pearson PJ, Schaff HV, Vanhoutte PM. Long-term impairment of endothelium-dependent relaxations to aggregating platelets after reperfusion injury in canine coronary arteries. Circulation 1990;81:1921-7.

18. Dignan RJ, Dyke CM, Abd-Elfattah AS, et al. Coronary artery endothelial cell and smooth muscle dysfunction after global myocardial ischemia. Ann Thorac Surg 1992;53:311-7.

19. Viehman GE, Xin-Liang MA, Lefer DJ, et al. Time course of endothelial dysfunction and myocardial injury during coronary arterial occlusion. Am J Physiol 1991;261:874-81. 